\title{
ACRL Nominees for Offices 1968/69
}

Vice President (President-Elect)

Page Ackerman, University of California, Los Angeles

Philip J. McNiff, Boston Public Library

Directors-at-Large (1968/72) (two to be elected, one from each bracket)

Frances Kennedy, Oklahoma City University

David Weber, Stanford University, Stanford, Calif.

J. Richard Blanchard, University of California, Davis

Norman Tanis, Kansas State College of Pittsburg

\section{College Libraries Section}

Vice Chairman (Chairman-Elect)

Donald Hendricks, Sam Houston State College, Huntsville, Tex.

John E. Scott, West Virginia State College, Institute, W. Va.

\section{Secretary}

Martha A. Connor, Swarthmore College, Swarthmore, $\mathrm{Pa}$.

Lydia M. Newland, Sweet Briar College, Sweet Briar, Va.

\section{Junior College Libraries Section}

Vice Chairman (Chairman-Elect)

Richard L. Ducote, College of Dupage, Glen Ellyn, Ill.

Hal C. Stone, Los Angeles City College

Secretary

Mrs. Mayrelee Newman, El Centro College, Dallas, Tex.

David L. Reich, Monroe County Community College, Monroe, Mich.

\section{Rare Books Section}

Vice Chairman (Chairman-Elect)

Robert Adelsperger, University of Illinois, Chicago Circle Campus

Donald Engley, Trinity College, Hartford, Conn.

\section{Secretary}

Mrs. Neda Westlake, University of Pennsylvania, Philadelphia

Mrs. Mildred Selle, University of Miami, Coral Gables, Fla.

\section{Subject Specialists Section}

\section{Vice Chairman (Chairman-Elect)}

Clifford R. Johnson, Project URBANDOC, New York

Marcia J. Miller, United States Department of Labor, Washington, D.C.

\section{Agriculture and Biological Sciences SUBSECTION}

Vice Chairman (Chairman-Elect)

J. Richard Blanchard, University of California, Davis

Howard Rovelstad, University of Maryland, College Park

\section{Secretary}

Mrs. Theodora Andrews, Purdue University, Lafayette, Ind.

Anna E. Dougherty, National Institutes of Health, Bethesda, Md.

\section{Art Subsection}

Vice Chairman (Chairman-Elect)

Wolfgang M. Freitag, Harvard University, Cambridge, Mass.

Elizabeth Halbe, Cleveland Museum of Art

Law and Political Science Subsection

Vice Chairman (Chairman-Elect)

Gerard Magavero, University of Mississippi Law School, University, Miss.

Roy Mersky, School of Law, University of Texas, Austin

Member-At-Large

Eleanor Tafel, Free Library of Philadelphia

Ludwik Teclaff, School of Law, Fordham University, New York

\section{Slavic and East European Subsection}

Vice Chairman (Chairman-Elect)

Eugene Petriwsky, University of Colorado, Boulder

Joseph A. Placek, University of Michigan, Ann Arbor

Member-At-Large

Boris I. Gorokhoff, Library of Congress, Washington, D.C.

Ray R. Suput, Case Western Reserve University, Cleveland

\section{University Libraries Section}

Vice Chairman (Chairman-Elect)

Roscoe Rouse, Oklahoma State University, Stillwater

Louis Vagianos, Dalhousie University, Halifax, N.S.

\section{Secretary}

Roy L. Kidman, University of California, Riverside

William V. Nash, University of Washington, Seattle 